\title{
Relativistic stellar oscillations treated as an initial value problem
}

\author{
S Kind, J Ehlers and B G Schmidt \\ Max-Planck-Institut für Astrophysik, Karl-Schwarzschild-Strasse 1, W-8046 Garching bei \\ Mïnchen, Federal Republic of Germany
}

Received 23 August 1992, in final form 17 May 1993

\begin{abstract}
The linearized Einstein equations for a static, spherically symmetric fluid ball and its empty surroundings are considered. It is shown that, given initial data obeying the constraints, there exists a unique solution, which describes the motion of the perturbed fluid and the gravitational waves propagating inside and outside the fiuid ball. The physical junction conditions for the boundary of the ball suffice to determine the evolution inside and outside of the ball in terms of initial values. The equation of state is assumed smooth and such that the density and the speed of sound remain positive for vanishing pressure.
\end{abstract}

PACS numbers: $0230,0420,0430$

\section{Introduction}

The theory of small adiabatic, non-radial perturbations of a star in hydrostatic equilibrium within the framework of general relativity was initiated by Thorne and Campolattaro in 1967 [1]. There, and in all of the following investigations (see [2] for a fairly complete list of references) up to the very recent ones by Chandrasekhar and Ferrari [3], harmonic analysis in time was immediately invoked. This leads, together with an expansion in spherical harmonics, to a system of ordinary differential equations, which was used mainly to determine the 'quasinormal modes' of the system (see [9] for a discussion of quasinormal modes). The mathematical question concerning the existence of solutions of the full timedependent equations was apparently never asked.

For radial oscillations the quasilinear problem has been treated in [13]. The analogous problem for non-radial oscillations appears to be beyond the reach of present mathematics; therefore we have to be content with a linearized treatment.

In spite of the fact that the equations are linear, one has a non-trivial problem for the following reason. On the background star one has a system of equations for the fluid perturbations coupled to the gravitational wave perturbations. Outside the star there are just the gravitational perturbations. On physical grounds one expects that Cauchy data at some time $t=0$, together with the general physical junction conditions, should determine a unique solution.

We can, for example, consider a case in which at $t=0$ there is just a gravitational wave outside the star moving towards it. If the gravitational wave impinges on the star it will be partly reflected, and partly enter the star and excite motions of the star which in turn will emit gravitational radiation. Such a solution should be completely determined by the data for the gravitational wave outside the star at $t=0$. There is no room for free data at the 
boundary. Just general junction conditions, as for example that the pressure vanishes at the boundary of the star, should make the evolution unique.

Similarly, if we disturb just the equilibrium density distribution, we expect that the matter will start to move and emit gravitational waves. Again, there should be a unique solution for such initial data.

The main purpose of this paper is to show that this is actually true for background models with positive density and positive velocity of sound at the boundary. The case in which the density and the velocity of sound vanish at the boundary can probably be treated by the same method.

In section 2 we formulate the field equations using the Regge-Wheeler gauge which removes all gauge freedom. We describe a formulation of the equations developed by Saskia Kind [4], in which there are three basic unknown variables, two linearized metric coefficients and the density perturbation. The three functions satisfy coupled linear wave equations; the characteristics of the metric quantities are the null cones and, for the density, the sound cones of the background spacetime.

Besides these evolution equations there are constraints which turn out to be compatible with the evolution.

This very useful separation of the equations into propagation and constraint equations is lost if one performs harmonic analysis in time. Then all equations contain only spatial derivatives and there are many choices of the basic unknowns. For example, Chandrasekhar and Ferrari use variables such that the matter perturbations are completely eliminated in favour of just metric perturbations from their fundamental system of equations. We think that a clearer physical picture emerges if we have the 'physical degrees of freedom'-two linearized metric coefficients for the gravitational waves and the perturbed density for the hydrodynamics-present.

In section 3 we analyse more abstractly systems of equations in which a different number of unknowns is coupled at a boundary. The method of characteristics, the key tool for $1+1$ hyperbolic systems, is used to find conditions at the boundary which make the Cauchy problem well posed.

Section 3 is the essential one. We demonstrate that the junction condition, the continuity of the first and second fundamental forms of the boundary hypersurface, contains enough information to make the Cauchy problem well posed. This result, together with an existence theorem proved in section 5, gives solutions in a neighbourhood of the boundary.

In section 6 we outline the construction of global solutions. To avoid a discussion of the polar coordinate singularity, we patch global (in space) solutions together, using a general existence theorem for linearized hydrodynamics near the centre. This way one obtains, for each spherical harmonic, a solution for all $r$ in some time interval. Since the equations are linear with bounded, time independent coefficients, this solution exists for all times and, for data with compact support, grows at most exponentially in time.

We want to finish this introduction by comparing briefly the 'time dependent' and 'time independent' treatment of oscillations. The actual solution of the physical problem, the motion of the fluid and the perturbed gravitational field, is time dependent. (This is also true for any real scattering process described in quantum mechanics.) If the coefficients of the linear equations do not depend on time, one can obtain a large class of solutions as a superposition of solutions with harmonic time dependence. In the time dependent problem a particular solution is determined by initial data for the fluid (or in quantum mechanics for the initial state). The boundary conditions one has to impose in the time independent treatment- outgoing waves at infinity"-can only be derived and understood as a consequence of the time-dependent formulation. The decay properties in time determine 
whether Fourier or Laplace transformation in time is possible. By considering just the time independent equations one loses important insight into the physical and mathematical nature of the solutions.

\section{Basic variables and equations}

The linearly perturbed metric describing axisymmetric even parity modes of 'angular momentum $l \geqslant 2$ ' of an oscillating perfect fluid in Regge-Wheeler gauge is

$$
\begin{gathered}
\mathrm{d} s^{2}=-c^{2} \mathrm{e}^{2 \Phi}\left(1-2 f P_{l}\right) \mathrm{d} t^{2}+\mathrm{e}^{2 \Lambda}\left(1+2 f P_{l}\right) \mathrm{d} r^{2}+2 c e^{\Phi+\Lambda} h P_{l} \mathrm{~d} t \mathrm{~d} r \\
+r^{2}\left(\mathrm{~d} \theta^{2}+\sin ^{2} \theta \mathrm{d} \phi^{2}\right)\left(1+2 g P_{l}\right)
\end{gathered}
$$

where the $(\theta, \theta)$ field equation has already been used to express the perturbation of $g_{t}$ in terms of that of $g_{r r} . f, g, h$ are functions of $(t, r)$ which denote the linearized perturbation of a static fluid solution determined by the functions $\Phi(r), \Lambda(r)$ which are smooth except at the boundary (see below). The Regge-Wheeler gauge is characterized by the property that the perturbed spacetime metric is block diagonal and the metric of the group orbits is perturbed just by a conformal factor.

The perturbed fluid variables are

$$
\delta \rho=\sigma P_{l} \quad \delta u_{r}=\mathrm{e}^{\Lambda} V P_{l} \quad \delta u_{\theta}=r W P_{\theta} .
$$

The time component of the velocity perturbation can be determined from the normalization of the 4-velocity. $V, W$ and $\sigma$ are again functions of $(r, t)$. The Regge-Wheeler gauge determines $f, g, h, \sigma, V, W$ uniquely $(l \geqslant 2)$.

We assume that the background model describes a fluid ball of finite radius $r_{0}$, with the property that the density and the velocity of sound are positive at the boundary. This is true if the equation of state $\rho(p)$ satisfies $\rho(0)=\rho_{0}>0$ and $\mathrm{d} p / \mathrm{d} \rho=: w^{2}>0$ [5]. Then, $\Phi$, $\Phi^{\prime}$ and $\Lambda$ are continuous at the boundary.

The linearized field equations $\delta R_{a b}=\delta\left(T_{a b}-\frac{1}{2} g_{a b} T_{c}^{c}\right.$ ) are (using the notation ${ }^{\circ}=\partial / \partial t$, $'=\partial / \partial r, \lambda=l(l+1))$

$\phi \phi$-component:

$$
\begin{aligned}
\left.c^{-2} \mathrm{e}^{2(\Lambda-\Phi}\right) \ddot{g}- & g^{\prime \prime}-g^{\prime}\left(\Phi^{\prime}-\Lambda^{\prime}+\frac{4}{r}\right)+\frac{2}{r} f^{\prime}+(f-g) \frac{2}{r}\left(\Phi^{\prime}-\Lambda^{\prime}+\frac{1}{r}\right) \\
& +\mathrm{e}^{2 \Lambda} \frac{\lambda}{r^{2}} g-\frac{1}{r c} \mathrm{e}^{(\Lambda-\Phi)} \dot{h} \\
= & \frac{4 \pi G}{c^{4}} \mathrm{e}^{2 \Lambda}\left(\sigma\left(c^{2}-w^{2}\right)+2 g\left(c^{2} \rho-p\right)\right)
\end{aligned}
$$

$r \theta$-component:

$$
(2 c)^{-1} \mathrm{e}^{(\Lambda-\Phi)} \dot{h}+g^{\prime}-f^{\prime}-2 \Phi^{\prime} f=0
$$

rr-component:

$$
\begin{aligned}
c^{-2} \mathrm{e}^{2(\Lambda-\Phi)} \ddot{f}+ & f^{\prime \prime}-2 g^{\prime \prime}+f^{\prime}\left(3 \Phi^{\prime}-\Lambda^{\prime}+\frac{2}{r}\right)+2 g^{\prime}\left(\Lambda^{\prime}-\frac{2}{r}\right)+\mathrm{e}^{2 \Lambda} \frac{\lambda}{r^{2}} f \\
& -c^{-1} \mathrm{e}^{(\Lambda-\Phi)}\left(\dot{h}^{\prime}+\Phi^{\prime} \dot{h}\right) \\
& =\frac{4 \pi G}{c^{4}} \mathrm{e}^{2 \Lambda}\left(\sigma\left(c^{2}-w^{2}\right)+2 f\left(c^{2} \rho-p\right)\right)
\end{aligned}
$$


$t t$-component:

$$
\begin{aligned}
c^{-2} \mathrm{e}^{2(\Lambda-\Phi)}(2 \ddot{g} & +\ddot{f})-f^{\prime \prime}-f^{\prime}\left(3 \Phi^{\prime}-\Lambda^{\prime}+\frac{2}{r}\right)+2 g^{\prime} \Phi^{\prime}+\mathrm{e}^{2 \Lambda} \frac{\lambda}{r^{2}} f \\
& +c^{-1} \mathrm{e}^{(\Lambda-\Phi)}\left(\dot{h}^{\prime}+\dot{h}\left(\Phi^{\prime}+\frac{2}{r}\right)\right) \\
= & \frac{4 \pi G}{c^{4}} \mathrm{e}^{2 \Lambda}\left(\sigma\left(c^{2}+3 w^{2}\right)+2 f\left(c^{2} \rho+3 p\right)\right)
\end{aligned}
$$

$r$-component:

$$
\begin{aligned}
Z_{\mathrm{l}}:=c^{-1} \mathrm{e}^{(\Lambda-\Phi)} & \left(\dot{g}^{\prime}-\dot{g}\left(\Phi^{\prime}-\frac{1}{r}\right)-\frac{1}{r} \dot{f}\right)-\mathrm{e}^{2 \Lambda} \frac{\lambda}{4 r^{2}} h \\
& -\frac{4 \pi G}{c^{4}} \mathrm{e}^{2 \Lambda}\left(c^{-1} V-h\right)\left(c^{2} \rho+p\right)=0
\end{aligned}
$$

$t \theta$-component:

$$
Z_{2}:=\frac{1}{2} c^{-1} \mathrm{e}^{(\Lambda-\Phi)}(\dot{f}+\dot{g})-\frac{1}{4}\left(h^{\prime}+2 \Phi^{\prime} h\right)-\frac{4 \pi G}{c^{4}} \mathrm{e}^{\Lambda} r c^{-1} W\left(c^{2} \rho+p\right)=0 .
$$

The linearized equations of motion for the fluid are

$t$-component:

$$
\begin{aligned}
c^{-1} \mathrm{e}^{(\Lambda-\Phi)}\left(c^{2} \dot{\sigma}\right. & \left.+\left(c^{2} \rho+p\right)(\dot{f}+2 \dot{g})\right)-\mathrm{e}^{\Lambda} \frac{\lambda}{r}\left(c^{2} \rho+p\right) c^{-1} W \\
& +\left(c^{2} \rho+p\right)\left(c^{-1} V^{\prime}-h^{\prime}+\left(c^{-1} V-h\right)\left(\Phi^{\prime}+\frac{2}{r}-\Phi^{\prime} c^{2} w^{-2}\right)\right)=0
\end{aligned}
$$

$r$-component:

$\left(c^{2} \rho+p\right)\left(c^{-2} \mathrm{e}^{(\Lambda-\Phi)} \dot{V}-f^{\prime}\right)+\sigma\left(c^{2}+w^{2}\right) \Phi^{\prime}+\left(\sigma w^{2}\right)^{\prime}=0$

$\theta$-component:

$$
\left(c^{2} \rho+p\right)\left(c^{-2} \mathrm{e}^{-\Phi} r \dot{W}-f\right)+\sigma w^{2}=0 .
$$

There are various possibilities in choosing a set of unknowns. We consider first the equations on the fluid ball of the background solution and proceed as Saskia Kind in [4] to derive a system for $f, g$ and a 'density variable'

$$
H:=\left(c^{2} \rho+p\right)^{-1} \sigma w^{2} .
$$

The time derivative of (2.9) gives an equation for $\ddot{\sigma}$ in which we eliminate $\ddot{f}, \ddot{g}, \dot{h}, \dot{V}, \dot{W}$. Rewriting this equation for $H$ gives the wave equation

$$
\begin{aligned}
-w^{-2} \mathrm{e}^{2(\Lambda-\Phi)} \ddot{H} & +H^{\prime \prime}+\left(\left(2-c^{2} w^{-2}\right) \Phi^{\prime}-\Lambda^{\prime}+\frac{2}{r}\right) H^{\prime}-\mathrm{e}^{2 \Lambda} \frac{\lambda}{r^{2}} H \\
& +\Phi^{\prime}\left(1-c^{2} w^{-2}\right)\left(f^{\prime}-2 g^{\prime}\right)-4 \Phi^{\prime} \Phi^{\prime} c^{2} w^{-2} f \\
& +\frac{4 \pi G}{c^{4}} \mathrm{e}^{2 \Lambda}\left(\left(c^{2} \rho+p\right)\left(3+c^{2} w^{-2}\right) H+2\left(c^{2} \rho+3 p\right) f\right)=0
\end{aligned}
$$


with sound characteristics, in which some 'source terms' are due to gravitational waves.

By eliminating $\dot{h}$ from (2.3) and (2.5) using (2.4) we obtain two wave equations for $f$ and $g$ with null characteristics

$$
\begin{gathered}
-c^{-2} \mathrm{e}^{2(\Lambda-\Phi)} \ddot{g}+g^{\prime \prime}+g^{\prime}\left(\Phi^{\prime}-\Lambda^{\prime}+\frac{2}{r}\right)-\mathrm{e}^{2 \Lambda} \frac{\lambda}{r^{2}} g+g \frac{2}{r}\left(\Phi^{\prime}-\Lambda^{\prime}+\frac{1}{r}\right)+f \frac{2}{r}\left(\Phi^{\prime}+\Lambda^{\prime}-\frac{1}{r}\right) \\
+\frac{4 \pi G}{c^{4}} \mathrm{e}^{2 \Lambda}\left(\left(c^{2} \rho+p\right)\left(c^{2} w^{-2}-1\right) H+2\left(c^{2} \rho-p\right) g\right)=0
\end{gathered}
$$

and

$$
\begin{aligned}
-c^{-2} \mathrm{e}^{2(\Lambda-\Phi)} \ddot{f} & +f^{\prime \prime}+f^{\prime}\left(5 \Phi^{\prime}-\Lambda^{\prime}-\frac{2}{r}\right)-\mathrm{e}^{2 \Lambda} \frac{\lambda}{r^{2}} f-4 g^{\prime}\left(\Phi^{\prime}-\frac{1}{r}\right) \\
& +4 f\left(\Phi^{\prime \prime}+2 \Phi^{\prime} \Phi^{\prime}-\Phi^{\prime} \Lambda^{\prime}\right) \\
& +\frac{4 \pi G}{c^{4}} \mathrm{e}^{2 \Lambda}\left(\left(c^{2} \rho+p\right)\left(c^{2} w^{-2}-1\right) H+2\left(c^{2} \rho-p\right) f\right)=0
\end{aligned}
$$

If we add (2.5) and twice (2.3) to (2.6) all time derivatives cancel and we obtain

$$
\begin{aligned}
Z:=g^{\prime \prime}-g^{\prime}\left(\Lambda^{\prime}-\frac{3}{r}\right)-\frac{1}{r} f^{\prime}-\mathrm{e}^{2 \Lambda} \frac{\lambda}{2 r^{2}}(g+f)+(g-f) \frac{1}{r}\left(\Phi^{\prime}-\Lambda^{\prime}+\frac{1}{r}\right) \\
+\frac{4 \pi G}{c^{4}} \mathrm{e}^{2 \Lambda}\left(\left(c^{2} \rho+p\right) c^{2} w^{-2} H+\left(c^{2} \rho-p\right) g+\left(c^{2} \rho+p\right) f\right)=0 .
\end{aligned}
$$

The equations (2.14)-(2.16) and (2.4) are equivalent to the equations (2.3)-(2.6). The equations (2.13)-(2.15) form a system of wave equations for the functions $f, g, H$. Because of the constraint (2.16) we cannot choose the data freely. A lengthy calculation shows that sufficiently differentiable solutions of $(2.13)-(2.15)$ (it is sufficient that $H$ is of class $C^{2}$ and $f, g$ are of class $C^{4}$ ) satisfy

$$
\begin{aligned}
-c^{-2} e^{2\lfloor\Lambda-\Phi)} \ddot{Z} & +Z^{\prime \prime}+Z^{\prime}\left(5\left(\Phi^{\prime}-\Lambda^{\prime}\right)+\frac{2}{r}\right)-e^{2 \Lambda} \frac{\lambda}{r^{2}} Z \\
& +2 Z\left(\Phi^{\prime \prime}-\Lambda^{\prime \prime}+3\left(\Phi^{\prime}-\Lambda^{\prime}\right)^{2}+\frac{4}{r} \Phi\right)=0 .
\end{aligned}
$$

Hence if $Z, \dot{Z}$ vanish initially and if $Z$ vanishes at the boundary, $(2.16)$ is satisfied in the domain of determinancy of these (trivial) data.

Having found $f, g, H$, equation (2.4) is an ordinary differential equation for $h$ which can be solved if initial data are given for $h$.

The remaining field equations (2.7) and (2.8) serve to define $V, W$. If all field equations are satisfied, the 'equations of motion' (2.9)-(2.11) follow from the Bianchi identities.

Outside matter we have to proceed differently because (2.7) and (2.8) are now constraints. Solutions of (2.14), (2.15) and (2.4) (with $H=0$ ) satisfy

$$
\begin{aligned}
& c^{-1} \mathrm{e}^{(\Lambda-\Phi)} \dot{Z}_{1}=Z^{\prime}+4 \Phi^{\prime} Z \\
& c^{-1} \mathrm{e}^{(\Lambda-\Phi)} \dot{Z}_{2}=Z .
\end{aligned}
$$


Hence these constraints propagate provided $Z$ vanishes identically.

We have thus shown that the local Cauchy problem consists of three linear wave equations inside the body and two wave equations outside. Hence local $C^{\infty}$ solutions of the field equations for the interior, respectively exterior, problem exist.

Our goal is to demonstrate the existence of solutions for the whole problem, the interior as well as the exterior, which are determined just by Cauchy data at some instant $t=0$ and junction conditions at the boundary.

We use a general existence theorem for linearized fluid solutions to obtain a local solution near the centre where our equations are singular because of the use of polar coordinates. If we prescribe axisymmetric even parity data the solution will also have this symmetry.

The only complicated part is a region around the boundary of the star. The interior equations need some information about infalling gravitational waves at the surface of the star such that a unique solution is determined. Similarly for the exterior region. In the next sections we will see that Cauchy data alone are sufficient to determine a solution near the boundary.

Finally we list two equations without second space derivatives which we will need in section 4 for the formulation of the junction conditions. They follow from $(2.14)-(2.16)$ : from (2.14) and (2.16) we eliminate $g^{\prime \prime}$ and obtain

$$
\begin{aligned}
c^{-2} \mathrm{e}^{(\Lambda-\Phi)} \ddot{g}-g^{\prime} & \left(\Phi^{\prime}-\frac{1}{r}\right)-\frac{1}{r} f^{\prime}+\mathrm{e}^{2 \Lambda} \frac{\lambda}{2 r^{2}}(g-f) \\
& -g\left(\frac{2}{r} \Phi^{\prime}+\frac{1}{r^{2}}\right)-f\left(\frac{2}{r} \Phi^{\prime}-\frac{1}{r^{2}}\right)+\frac{4 \pi G}{c^{4}} \mathrm{e}^{2 \Lambda}\left(\left(c^{2} \rho+p\right) H+2 p g\right)=0 .
\end{aligned}
$$

To eliminate $f^{\prime \prime}$ in (2.15) we differentiate (2.16) with respect to $r$ and insert it into (2.15). The terms with $g^{\prime \prime}$ and $g^{\prime \prime \prime}$ which appear are eliminated using (2.14) and its $r$ derivative. The result is

$$
\begin{aligned}
c^{-2} \mathrm{e}^{(\Lambda-\Phi)}\left(\ddot{g}^{\prime}-\right. & \left.\ddot{g}\left(\Phi^{\prime}-\frac{1}{r}\right)-\frac{1}{r} \ddot{f}\right)-\mathrm{e}^{2 \Lambda} \frac{\lambda}{2 r^{2}}\left(f^{\prime}-g^{\prime}+2 \Phi^{\prime} f\right) \\
& +\frac{4 \pi G}{c^{4}} \mathrm{e}^{2 \Lambda}\left(c^{2} \rho+p\right)\left(H^{\prime}+f^{\prime}-2 g^{\prime}+4 \Phi^{\prime} f\right)=0 .
\end{aligned}
$$

\section{Systems of $1+1$ wave equations}

Existence and uniqueness of solutions for the system of wave equations derived in the last section can be established by the method of characteristics. We need a theorem for the inside and outside systems which are coupled by some boundary conditions. In this section we analyse the conditions which are needed at the boundary $r=r_{0}$ to make the Cauchy problem well posed.

For $a<r<r_{0}$ we have a system for $U_{k}(t, r), V_{j}(t, r)$

$$
\begin{array}{ll}
-\ddot{U}_{k}+a_{k}^{2} U_{k}^{\prime \prime}=F\left(\dot{U}_{k}, U_{k}^{\prime}, U_{k}, V_{j}\right) & k=1, \ldots, p, a_{k}>0 \\
\dot{V}_{j}=G\left(\dot{U}_{k}, U_{k}^{\prime}, U_{k}, V_{j}\right) & j=1, \ldots, l .
\end{array}
$$


In the last section we saw that we have to solve wave equations. Later it will tum out that to control the conditions $(2.16), Z=0$, at the boundary we have to differentiate our basic system with respect to $t$. This is why we introduced the functions $V_{j}$ in the equations above.

Similarly for $r_{0}<r$

$$
\begin{array}{ll}
-\dot{\bar{U}}_{k}+\bar{a}_{k}^{2} \bar{U}_{k}^{\prime \prime}=\bar{F}\left(\dot{\bar{U}}_{k}, \bar{U}_{k}^{\prime}, \bar{U}_{k}, \bar{V}_{j}\right) & k=1, \ldots, \bar{p}, \quad \bar{a}_{k}>0 \\
\dot{\bar{V}}_{j}=\bar{G}\left(\dot{\bar{U}}_{k}, \bar{U}_{k}^{\prime}, \bar{U}_{k}, \bar{V}_{j}\right) & j=1, \ldots, \bar{l} .
\end{array}
$$

The functions $F, \bar{F}, G, \bar{G}$ are linear in their arguments with coefficients depending only on $r$. We assume that $p \geqslant \bar{p}$.

We can rewrite the wave equations in characteristic form as follows

$$
\begin{aligned}
& \left(\partial_{t}+a_{k} \partial_{r}\right)\left(-\dot{U}_{k}+a_{k} U_{k}^{\prime}\right)=+a_{k} a_{k}^{\prime} U_{k}^{\prime}+F\left(\dot{U}_{k}, U_{k}^{\prime}, U_{k}, V_{j}\right) \\
& \left(\partial_{t}-a_{k} \partial_{r}\right)\left(\dot{U}_{k}+a_{k} U_{k}^{\prime}\right)=-a_{k} a_{k}^{\prime} U_{k}^{\prime}-F\left(\dot{U}_{k}, U_{k}^{\prime}, U_{k}, V_{j}\right)
\end{aligned}
$$

In terms of the functions

$$
A_{k}:=-\dot{U}_{k}+a_{k} U_{k}^{\prime} \quad B_{k}:=\dot{U}_{k}+a_{k} U_{k}^{\prime}
$$

which propagate along the characteristics, the system is equivalent to

$$
\begin{aligned}
& \left(\partial_{t}+a_{k} \partial_{r}\right) A_{k}=+a_{k}^{\prime} \frac{1}{2}\left(A_{k}+B_{k}\right)+F\left(\frac{1}{2}\left(B_{k}-A_{k}\right), \frac{1}{2 a_{k}}\left(B_{k}+A_{k}\right), U_{k}, V_{J}\right) \\
& \left(\partial_{t}-a_{k} \partial_{r}\right) B_{k}=-a_{k}^{\prime} \frac{1}{2}\left(A_{k}+B_{k}\right)-F\left(\frac{1}{2}\left(B_{k}-A_{k}\right), \frac{1}{2 a_{k}}\left(B_{k}+A_{k}\right), U_{k}, V_{j}\right) \\
& \dot{U}_{k}=\frac{1}{2}\left(B_{k}-A_{k}\right) \\
& \dot{V}_{j}=G\left(\frac{1}{2}\left(B_{k}-A_{k}\right), \frac{1}{2 a_{k}}\left(B_{k}+A_{k}\right), U_{k}, V_{j}\right) \\
& U_{k}^{\prime}=\frac{1}{2 a_{k}}\left(B_{k}+A_{k}\right) .
\end{aligned}
$$

The last equation is a constraint, and the time evolution equations of (3.6) imply (provided there is sufficient differentiability)

$$
\partial_{t}\left(U_{k}^{\prime}-\frac{1}{2 a_{k}}\left(B_{k}+A_{k}\right)\right)=0 .
$$

Hence if $U_{k}^{\prime}-\left(1 / 2 a_{k}\right)\left(B_{k}+A_{k}\right)$ vanishes initially it vanishes for all time and a solution of (3.6) is also a solution of (3.1).

The functions $V_{j}$ propagate along the curves $r=$ constant and the functions $A_{k}, B_{k}$ along the integral curves

$$
r(t)=r_{k}^{+}(t ; \bar{t}, \bar{r}) \quad \text { and } \quad r(t)=r_{k}^{-}(t ; \bar{t}, \bar{r})
$$




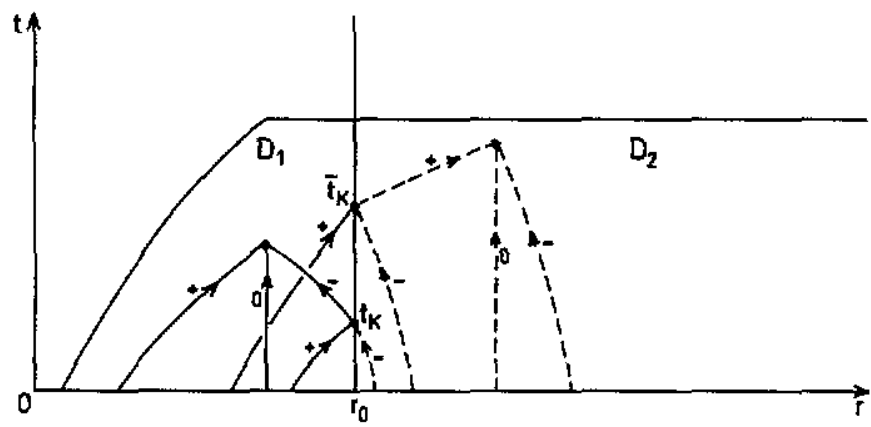

Figure 1. The domain $D=D_{1} \cup D_{2}$ and the boundary $r=r_{0}$ separating $D_{1}$ and $D_{2}$. The functions $A_{k}, B_{k}, Z_{m}$ in $D_{1}$ are transported along the outgoing ( + ), ingoing ( - ) and stationary (0) characteristics, respectively; the corresponding statement holds for $\tilde{A}_{k}, \bar{B}_{k}, \bar{Z}_{m}$ in $D_{2}$. Some characteristics hit the boundary at times $t_{k}, \bar{i}_{k}$. This requires conditions like $(3.20)-(3.22)$ in order that all functions be determined solely by initial data at $t=0$. For simplicity only one characteristic of each type is shown.

of the vector fields $\partial_{t} \pm a_{k} \partial r$ through the point $(\bar{t}, \bar{r})$, i.e.

$$
r_{k}^{+}(\bar{t} ; \bar{t}, \bar{r})=\bar{r} \quad r_{k}^{-}(\bar{t} ; \bar{t}, \bar{r})=\bar{r}
$$

(see figure 1). Integrating along the characteristic curves we transform the system (3.6) into a system of linear integral equations. We consider the equations on a domain $D$ such that for $r<r_{0}$ all $A$-characteristics and for $r_{0}<r$ all $B$-characteristics intersect $t=0$. We denote the part of $D$ with $r<r_{0}$ as $D_{1}$ and the part with $r_{0}<r$ as $D_{2}$. To simplify the integral equations we write $\left(Z_{m}\right):=\left(U_{k}, V_{j}\right)$ and $\left(E_{n}\right):=\left(A_{k}, B_{k}, Z_{m}\right)$; then the system (3.6) reads

$$
\begin{aligned}
& \left(\partial_{t}+a_{k} \partial_{r}\right) A_{k}=M_{k}\left(r, E_{n}\right) \\
& \left(\partial_{t}-a_{k} \partial_{r}\right) B_{k}=N_{k}\left(r, E_{n}\right) \\
& \partial_{t} Z_{m}=P_{m}\left(r, E_{n}\right) .
\end{aligned}
$$

The functions $M_{k}, N_{k}, P_{m}$ are linear in $E_{n}$ with coefficients depending on $r$. We integrate from $t=0$ to $(t, r)$ and denote initial data by ${ }^{\wedge \prime}$, obtaining

$$
\begin{gathered}
Z_{m}(t, r)=\hat{Z}_{m}(r)+\int_{0}^{t} P_{m}\left(r, E_{n}(s, r)\right) \mathrm{d} s \\
A_{k}(t, r)=\hat{A}_{k}\left(r_{k}^{+}(0 ; t, r)\right)+\int_{0}^{t} \mathrm{~d} s\left[M_{k}\left(r, E_{n}(s, r)\right)\right]_{r=r_{k}^{+}(s ; t, r)} .
\end{gathered}
$$

For $B_{k}$ there are two possibilities. If the backwards characteristic intersects $t=0$ we have

$$
{ }^{1} B_{k}(t, r)=\hat{B}_{k}\left(r_{k}^{-}(0 ; t, r)\right)+\int_{0}^{t} \mathrm{~d} s\left[N_{k}\left(r, E_{n}(s, r)\right)\right]_{r=r_{k}^{-}(s ; t, r)} .
$$

If, however, the characteristic intersects the line $\left(t, r_{0}\right)$ at the point $t_{k}$

$$
\left.{ }^{2} B_{k}(t, r)=B_{k}\left(t_{k}, r_{0}\right)\right)+\int_{t_{k}}^{t} \mathrm{~d} s\left[N_{k}\left(r, E_{n}(s, r)\right)\right]_{r=r_{k}^{-}(s ; t, r)} .
$$


On $D_{2}$, we proceed analogously and rewrite the system (3.2) as

$$
\begin{aligned}
& \left(\partial_{t}+\bar{a}_{k} \partial_{r}\right) \bar{A}_{k}=\bar{M}_{k}\left(r, \bar{E}_{n}\right) \\
& \left(\partial_{t}-\bar{a}_{k} \partial_{r}\right) \bar{B}_{k}=\bar{N}_{k}\left(r, \bar{E}_{n}\right) \\
& \partial_{t} \bar{Z}_{m}=\bar{P}_{m}\left(r, \bar{E}_{n}\right)
\end{aligned}
$$

The integration is as before

$$
\begin{gathered}
\bar{Z}_{m}(t, r)=\hat{\bar{Z}}_{m}(r)+\int_{0}^{t} \bar{P}_{m}\left(r, \bar{E}_{n}(s, r)\right) \mathrm{d} s \\
\bar{B}_{k}(t, r)=\hat{\bar{B}}_{k}\left(\bar{r}_{k}^{-}(0 ; t, r)\right)+\int_{0}^{t} \mathrm{~d} s\left[\bar{M}_{k}\left(r, \bar{E}_{n}(s, r)\right)\right]_{r=\bar{r}_{k}^{-}(s ; t, r)}
\end{gathered}
$$

Now there are two possibilities for $\bar{A}_{k}$. If the backwards characteristic intersects $t=0$ we have

${ }^{1} \bar{A}_{k}(t, r)=\hat{\bar{A}}_{k}\left(r_{k}^{+}(0 ; t, r)\right)+\int_{0}^{t} \mathrm{~d} s\left[\bar{N}_{k}\left(r, \bar{E}_{n}(s, r)\right)\right]_{r=\bar{n}_{k}^{+}(s ; t, r)}$.

If, however, the characteristic intersects the line $\left(t, r_{0}\right)$ at the point $\bar{t}_{k}$, then

$$
\left.{ }^{2} \bar{A}_{k}(t, r)=\bar{A}_{k}\left(\bar{t}_{k}, r_{0}\right)\right)+\int_{\bar{t}_{k}}^{t} \mathrm{~d} s\left[\bar{N}_{k}\left(r, \bar{E}_{n}(s, r)\right)\right]_{r=\bar{r}_{k}^{+}(s ; t, r)}
$$

The only information not supplied by the Cauchy data are the functions $B_{k}\left(t, r_{0}\right)$ and $\bar{A}_{k}\left(t, r_{0}\right)$ in (3.14) and (3.19), which are needed if the characteristics intersect the boundary line $r=r_{0}$. In order to obtain a system of integral equations for which only initial data are needed, we assume that at the boundary, for $1 \leqslant k \leqslant \tilde{p}$

$$
\begin{array}{ll}
B_{k}=\alpha A_{k}+\beta \bar{B}_{k}+K\left(Z_{m}, \bar{Z}_{\bar{m}}\right) & K \text { linear, } \quad \alpha, \beta=\text { constant } \\
\bar{A}_{k}=\bar{\alpha} \bar{B}_{k}+\bar{\beta} A_{k}+\bar{K}\left(Z_{m}, \bar{Z}_{\bar{m}}\right) & \bar{K} \text { linear, } \quad \bar{\alpha}, \bar{\beta}=\text { constant }
\end{array}
$$

and, if $p>\bar{p}$, for $\bar{p}+1 \leqslant \ell \leqslant p$

$$
B_{l}=\gamma A_{l}+D\left(Z_{m}, \bar{Z}_{\bar{m}}\right)
$$

We use these relations to express the missing values $\bar{A}_{k}\left(\bar{t}_{k}, r_{0}\right)$ and $B_{k}\left(t_{k}, r_{0}\right)$ in terms of the Cauchy data, using for the right-hand sides of (3.20)-(3.22) again (3.11), (3.12), (3.16), (3.17).

Now we have obtained a system of linear integral equations whose coefficients contain only the Cauchy data and the coefficients of the original differential equations. In section 5 we prove an existence and uniqueness theorem for this situation which provides $C^{k}$ solutions on the two domains if the coefficients of the differential equations are $C^{k+2}$ and the initial data are chosen appropriately. 


\section{The boundary conditions}

To linearize a one-parameter family of solutions describing an oscillating fluid ball together with its outside gravitational field we assume that there are coordinates in which the surface of the ball, which is generated by fluid world lines and at which the pressure vanishes [6], has the same coordinate values for all members of the family. One can, for example, take Gauss coordinates relative to the surface. Linearization implies that in this gauge the linearized pressure vanishes at the boundary and that the linearized first and second fundamental forms of the boundary are continuous. This is equivalent to the statement that in Regge-Wheeler gauge at $r=r_{0}$

$$
g_{1}=g_{2} \quad f_{1}=f_{2} \quad h_{1}=h_{2}
$$

where the indices 1,2 refer to the interior and exterior, respectively. Because of (2.4), the boundary condition for $h$ can be replaced by a continuity condition

$$
g-f \in C^{\prime} .
$$

The equations we derived in section 2 are; on the fluid ball

$$
\begin{aligned}
& -\ddot{f}_{1}+a_{1}^{2} f_{1}^{\prime \prime}=X_{1}\left(f_{1}, f_{1}^{\prime}, g_{1}^{\prime}, H\right) \\
& -\ddot{g}_{1}+a_{1}^{2} g_{1}^{\prime \prime}=Y_{1}\left(f_{1}, g_{1}, g_{1}^{\prime}, H\right) \\
& -\ddot{H}+b^{2} H^{\prime \prime}=L\left(f, f^{\prime}, g^{\prime}, H, H^{\prime}\right)
\end{aligned}
$$

and outside

$$
\begin{aligned}
& -\ddot{f}_{2}+a_{2}^{2} f_{2}^{\prime \prime}=X_{2}\left(f_{2}, f_{2}^{\prime}, g_{2}^{\prime}\right) \\
& -\ddot{g}_{2}+a_{2}^{2} g_{2}^{\prime \prime}=Y_{2}\left(f_{2}, g_{2}, g_{2}^{\prime}\right) .
\end{aligned}
$$

Hence we have the situation treated in the last section. $f, g$ are defined on both sides of the boundary and $H$ is defined only on the fluid ball. On physical grounds we expect that Cauchy data together with the junction conditions (4.1) should determine a unique solution. Hence we have to investigate whether the conditions (4.1) together with the field equations contain sufficient information to obtain conditions in the form of (3.20)-(3.22) considered in the last section.

To obtain a solution of the field equations we have to satisfy not only the above system, but also the condition $Z \equiv 0$. As discussed in section 2 this is implied by the field equations if $Z=0$ for the data and at the boundary. Hence we have to satisfy (2.19) at the boundary

$$
\begin{aligned}
c^{-2} \mathrm{e}^{(\Lambda-\Phi)} \ddot{g}_{1}- & g_{1}^{\prime}\left(\Phi^{\prime}-\frac{1}{r}\right)-\frac{1}{r} f_{1}^{\prime}+\mathrm{e}^{2 \Lambda} \frac{\lambda}{2 r^{2}}\left(g_{1}-f_{1}\right)-g_{1}\left(\frac{2}{r} \Phi^{\prime}+\frac{1}{r^{2}}\right) \\
- & f_{1}\left(\frac{2}{r} \Phi^{\prime}-\frac{1}{r^{2}}\right)+\frac{4 \pi G}{c^{4}} \mathrm{e}^{2 \Lambda}\left(\left(c^{2} \rho+p\right) H+2 p g_{1}\right)=0
\end{aligned}
$$

and

$$
\begin{aligned}
c^{-2} \mathrm{e}^{(\Lambda-\Phi)} \ddot{g}_{2}- & g_{2}^{\prime}\left(\Phi^{\prime}-\frac{1}{r}\right)-\frac{1}{r} f_{2}^{\prime}+\mathrm{e}^{2 \Lambda} \frac{\lambda}{2 r^{2}}\left(g_{2}-f_{2}\right) \\
& -g_{2}\left(\frac{2}{r} \Phi^{\prime}+\frac{1}{r^{2}}\right)-f_{2}\left(\frac{2}{r} \Phi^{\prime}-\frac{1}{r^{2}}\right)=0 .
\end{aligned}
$$


Because of the second time derivatives we can use these conditions only if we use $\dot{g}$ as the unknown function. Differentiating the equations (4.4) and (4.7) for $g$ with respect to $t$ we obtain for

$$
z_{1}=\dot{g}_{1} \quad z_{2}=\dot{g}_{2}
$$

the equations

$$
\begin{aligned}
& -\ddot{z}_{1}+a_{1}^{2} z_{1}^{\prime \prime}=Y_{1}\left(\dot{f}_{1}, z_{1}, z_{1}^{\prime}, \dot{H}\right) \\
& -\ddot{z}_{2}+a_{2}^{2} z_{2}^{\prime \prime}=Y_{2}\left(\dot{f}_{2}, z_{2}, z_{2}^{\prime}\right) .
\end{aligned}
$$

Now the conditions (4.8) and (4.9) are of the form (3.20), (3.21), (we now use the notation from the last section and put $u_{2}=z$ )

$$
B_{2}=A_{2}+2 \dot{z}_{1} \quad \bar{A}_{2}=\bar{B}_{2}-2 \dot{z}_{2}
$$

where $\dot{z}$ is taken from (4.8), (4.9). To take care of $f^{\prime}, g^{\prime}$ in (4.8), (4.9), we have to treat $f^{\prime}, g^{\prime}, \dot{f}, \dot{g}$ as unknowns together with $z$, to form a system like (3.1).

Next we need conditions for $f$ and $H$. The comparison of (4.8) and (4.9) between the inside and outside gives at $r=r_{0}$ where $p^{\prime}=0$

$$
\Phi^{\prime}\left(g_{1}^{\prime}-g_{2}^{\prime}\right)=\frac{4 \pi G}{c^{2}} \mathrm{e}^{2 \Lambda} \rho H .
$$

Because of (4.2) we also have

$$
\Phi^{\prime}\left(f_{1}^{\prime}-f_{2}^{\prime}\right)=\frac{4 \pi G}{c^{2}} \mathrm{e}^{2 \Lambda} \rho H .
$$

From $(4.1),(4.14),(4.15)$ we derive

$$
\dot{f}_{1} \pm c^{2} \mathrm{e}^{2(\Phi-\Lambda)} f_{1}^{\prime}=\dot{f}_{2} \pm c^{2} \mathrm{e}^{2(\Phi-\Lambda)} f_{2}^{\prime} \pm H .
$$

These are conditions of the form (3.20), (3.21) needed for $f$ at the boundary. It remains to find a boundary condition for $H$. No relation between $\dot{H}$ and $H^{\prime}$ was found. However, comparing (2.20) from both sides at the boundary one finds, using (4.1)

$$
c^{-2} \mathrm{e}^{-2 \phi}\left(\ddot{g}_{1}^{\prime}-\ddot{g}_{2}^{\prime}\right)+\frac{4 \pi G}{c^{2}} \rho\left(H^{\prime}+f_{1}^{\prime}-2 g_{1}^{\prime}+4 \Phi^{\prime} f_{1}\right)=0 .
$$

A second time derivative of (4.14), inserted into (4.17), gives

$$
c^{-2} \mathrm{e}^{2(\Lambda-\phi)} \ddot{H}+\Phi^{\prime}\left(H^{\prime}+f_{1}^{\prime}-2 g_{1}^{\prime}+4 \Phi^{\prime} f_{1}\right)=0 .
$$

We can use this condition if we take $C:=\dot{H}$ as an unknown function instead of $H$. As $C$ is defined only for $a<r \leqslant r_{0}$ we need condition (3.22), and (4.18) allows us to calculate the ingoing quantity from the outgoing one. If we differentiate (4.6) with respect to time, a term $\dot{f}^{\prime}$ appears. Therefore we have finally also to use the time derivative of the equation for $f$ and use a time derivative of (4.16) at the boundary. 
Proceeding in this way we have finally to check that for the solution obtained, we have

$$
g_{1}=g_{2} \quad \text { and } \quad g_{1}^{\prime}-f_{1}^{\prime}=g_{2}^{\prime}-f_{2}^{\prime}
$$

This is established as follows: assuming (4.8), (4.9), (4.18), (4.16) and $f_{1}=f_{2}$ and comparing (4.18) from both sides gives $\left(\Delta a=a_{1}-a_{2}\right)$

$$
-c^{-2} \mathrm{e}^{2(\Lambda-\Phi)} \Delta \ddot{g}+\left(\Phi^{\prime}-\frac{1}{r}\right) \Delta\left(g^{\prime}-f^{\prime}\right)+\left(\frac{2}{r} \Phi^{\prime}+\frac{1}{r^{2}}-\mathrm{e}^{2 \Lambda} \frac{\lambda}{2 r^{2}}\right) \Delta g=0 .
$$

Comparing with $(2.20)$ gives

$\left.c^{-2} \mathrm{e}^{-2 \Phi}\left(\Delta \ddot{g}^{\prime}-\left(\Phi^{\prime}-\frac{1}{r}\right) \Delta \ddot{g}\right)-\frac{\lambda}{2 r^{2}}\right) \Delta\left(f^{\prime}-g^{\prime}\right)-\frac{4 \pi G}{c^{2}} \rho \frac{1}{c^{2} \Phi^{\prime}} \mathrm{e}^{2(\Lambda-\Phi)} \ddot{H}=0$.

Using (4.16) we replace $\ddot{H}$ by $\Delta \ddot{f}^{\prime}$ and get

$c^{-2} \mathrm{e}^{-2 \Phi}\left(\Delta \ddot{g}^{\prime}-\Delta \ddot{f}^{\prime}-\left(\Phi^{\prime}-\frac{1}{r}\right) \Delta \ddot{g}\right)-\frac{\lambda}{2 r^{2}} \Delta\left(f^{\prime}-g^{\prime}\right)=0$.

Equations (4.20) and (4.22) form a linear, homogeneous, ordinary differential system for $\Delta g$ and $\Delta\left(f^{\prime}-g^{\prime}\right)$ as functions of $t$. If these quantities and their first time derivatives vanish at $t=0$, they are identically zero.

We have shown in this section that the physical junction conditions (4.1) provide just enough information to rewrite the equations as integral equations in which only the Cauchy data appear.

\section{An existence theorem}

In section 3 we obtained a system of linear integral equations. Now we will show the existence of solutions, using the method outlined in $[7,8] . \quad\left(\left(Z_{m}\right):=\left(U_{k}, V_{j}\right)\right.$ and $\left(E_{n}\right):=\left(A_{k}, B_{k}, Z_{m}\right)$, quantities with a $" \wedge$ are data at $t=0$.) It may be helpful in connection with the following calculations to look at figure 1 once more.

Within the star, $r<r_{0}$, we have

$$
\begin{gathered}
Z_{m}(t, r)=\hat{Z}_{m}(r)+\int_{0}^{t} P_{m}\left(r, E_{n}(s, r)\right) \mathrm{d} s \\
A_{k}(t, r)=\hat{A}_{k}\left(r_{k}^{+}(0 ; t, r)\right)+\int_{0}^{t} \mathrm{~d} s\left[M_{k}\left(r, E_{n}(s, r)\right)\right]_{r=r_{k}^{+}(s ; t, r)} .
\end{gathered}
$$

For $B_{k}$ there are two possibilities. If the backwards characteristic intersects $t=0$, we have

$$
{ }^{1} B_{k}(t, r)=\hat{B}_{k}\left(r_{k}^{-}(0 ; t, r)\right)+\int_{0}^{t} \mathrm{~d} s\left[N_{k}\left(r, E_{n}(s, r)\right)\right]_{r=r_{k}^{-}(s ; t, r)} .
$$

If, however, the characteristic intersects the line $\left(t, r_{0}\right)$ at the point $t_{k}$, we have

$$
\left.{ }^{2} B_{k}(t, r)=B_{k}\left(t_{k}, r_{0}\right)\right)+\int_{t_{k}}^{t} \mathrm{~d} s\left[N_{k}\left(r, E_{n}(s, r)\right)\right]_{r=r_{k}^{-}(s ; r, r)} .
$$


For $r>r_{0}$, outside the star

$$
\begin{gathered}
\bar{Z}_{m}(t, r)=\hat{\bar{Z}}_{m}(r)+\int_{0}^{t} \vec{P}_{m}\left(r, \bar{E}_{n}(s, r)\right) \mathrm{d} s \\
\vec{B}_{k}(t, r)=\hat{\bar{B}}_{k}\left(r_{k}^{-}(0 ; t, r)\right)+\int_{0}^{t} \mathrm{~d} s\left[\bar{M}_{k}\left(r, \bar{E}_{n}(s, r)\right)\right]_{r=\bar{r}_{k}^{-}(s ; f, r)} .
\end{gathered}
$$

Now there are two possibilities for $\bar{A}_{k}$. If the backwards characteristic intersects $t=0$, we have

$$
{ }^{1} \bar{A}_{k}(t, r)=\hat{\bar{A}}_{k}\left(r_{k}^{+}(0 ; t, r)\right)+\int_{0}^{t} \mathrm{~d} s\left[\bar{N}_{k}\left(r, \bar{E}_{n}(s, r)\right)\right]_{r=\tilde{r}_{k}^{+}(s: t, r)}
$$

If, however, the characteristic intersects the line $\left(t, r_{0}\right)$ at the point $\bar{t}_{k}$

$$
\left.{ }^{2} \bar{A}_{k}(t, r)=\hat{\bar{A}}_{k}\left(\bar{t}_{k}, r_{0}\right)\right)+\int_{\bar{t}_{k}}^{t} \mathrm{~d} s\left[\bar{N}_{k}\left(r, \bar{E}_{n}(s, r)\right)\right]_{r=\bar{r}_{k}^{+}(s ; t, r)} .
$$

Assume that at the boundary we impose conditions

$$
\begin{array}{ll}
B_{k}=\alpha A_{k}+\beta \bar{B}_{k}+K\left(Z_{m}, \bar{Z}_{\bar{m}}\right) & K \text { linear, } \quad \alpha, \beta=\text { constant } \\
\bar{A}_{k}=\bar{\alpha} \bar{B}_{k}+\bar{\beta} A_{k}+\bar{K}\left(Z_{m}, \bar{Z}_{\bar{m}}\right) & \bar{K} \text { linear, } \quad \bar{\alpha}, \bar{\beta}=\text { constant. }
\end{array}
$$

for $A, B, \bar{A}, \bar{B}$ defined on the appropriate side. (If some of the variables are defined only for $r \leqslant r_{0}$, we have to put $B=0$ in (5.9).) We use these relations to express the missing values $B_{k}\left(t_{k}, r_{1}\right)$ and $\bar{A}_{k}\left(\bar{t}_{k}, r_{1}\right)$ in terms of quantities which can be calculated from the Cauchy data.

If we insert the boundary conditions in (5.4), we obtain

$$
\begin{gathered}
{ }^{2} B_{k}(t, r)=\alpha A_{k}\left(t_{k}, r_{0}\right)+\beta \bar{B}_{k}\left(t_{k}, r_{0}\right)+K\left(Z_{m}\left(t_{k}, r_{0}\right), \bar{Z}_{\bar{m}}\left(t_{k}, r_{0}\right)\right. \\
+\int_{t_{k}}^{t} \mathrm{~d} s\left[N_{k}\left(r, E_{n}(s, r)\right)\right]_{r=r_{k}^{-}(s ; t, r)} .
\end{gathered}
$$

Similarly from (5.8)

$$
\begin{gathered}
{ }^{2} \bar{A}_{k}(t, r)=\bar{\alpha} \bar{B}_{k}\left(\bar{t}_{k}, r_{0}\right)+\bar{\beta} A_{k}\left(\bar{t}_{k}, r_{0}\right)+\bar{K}\left(Z_{m}\left(\bar{t}_{k}, r_{0}\right), \bar{Z}_{\bar{m}}\left(\bar{t}_{k}, r_{0}\right)\right) \\
+\int_{\bar{t}_{k}}^{t} \mathrm{~d} s\left[\bar{N}_{k}\left(r, \bar{E}_{n}(s, r)\right)\right]_{r=\bar{r}_{k}^{+}(s ; t, r)} .
\end{gathered}
$$

In (5.11) and (5.12) all the quantities at $\left(t_{k}, r_{0}\right)$ and $\left(\bar{t}_{k}, r_{0}\right)$ can be expressed in terms of $(5.1)-(5.3),(5.5)-(5.7)$. Now we have obtained a system of integral equations for the unknowns.

Solutions of such systems are obtained by iteration. Suppose differentiable functions $E_{n}^{(0)}$ are given satisfying the initial conditions. Inserting these functions in all the integrals and performing the integrations we obtain functions $E_{n}^{(1)}$, which again satisfy the initial conditions. Thus we generate a sequence $E_{n}^{(j)}$. The linear operator mapping $E_{n}^{(j)}$ to $E_{n}^{(j+i)}$ consists of a finite number of integrations. If we write

$$
\left.\|E\|=\max \left\{\mid E_{n}\right\},\left|\bar{E}_{n}\right|\right\} \quad \text { for } n=1, \ldots, N, \quad \bar{n}=1, \ldots, \bar{N} \quad \text { and } \quad(t, r) \in D
$$


we obtain

$$
\left\|{ }^{(j+1)} E-{ }^{(j)} E\right\| \leqslant \delta\left\|^{(j)} E-{ }^{(j-1)} E\right\| \times \text { constant. }
$$

The constant depends on the maximum of all the coefficients in the integrals and the number of those integrals; $\delta$ is the maximum of the lengths of the domains of integration, hence it is determined by the slopes of the characteristics and the domain $D$. We can make $\delta$ as small as we want, and therefore the sequence $\left\|E^{(j)}\right\|$ is a Cauchy sequence. This implies that the functions ${ }^{(j)} E(r, t)$ and ${ }^{(j)} \bar{E}(r, t)$ converge uniformly in $D$ to continuous functions, and these limits satisfy the integral equations.

On the characteristics through $\left(0, r_{0}\right)$ we have two expressions. We obtain for all iterates the same values, if the data satisfy the conditions $(5.9),(5.10)$ at $\left(0, r_{0}\right)$.

Moreover we have to show that the solution of the integral equations is differentiable, because only then have we obtained a solution of the differential equations. If all iterates are differentiable and if these derivatives converge in the maximum norm, then they converge to the derivative of the solution.

For the derivative with respect to $r$ we obtain by differentiating the expressions defining $E_{n t}^{(j)}$, an inequality as (5.14) if we include into the definition of $\|E\|$ also the derivatives of the unknown $E_{n}, \bar{E}_{m}$. Hence we obtain convergence of the $r$ derivative. The integral equations imply that we can differentiate in the direction of the characteristics, hence all functions are $C^{1}$.

Again there are two expressions for the derivatives along the characteristics through $\left(0, r_{0}\right)$ and we have to check that we obtain the same values. This will give further 'corner conditions' which guarantee the differentiability of the solution. Let us consider this in more detail.

Assume that we have a solution which is $C^{1}$ and satisfies the conditions (5.9), (5.10). Then there are two ways of calculating $B_{k}\left(0, r_{0}\right)$. From the data we know $B_{k}^{\prime}\left(0, r_{0}\right)$ and the differential equation gives the derivative in the direction of the characteristic. Hence we also obtain $\dot{B}_{k}\left(0, r_{0}\right)$. Alternatively we may start from the boundary condition (5.9) to calculate $\dot{B}_{k}\left(0, r_{0}\right)$. The condition that we obtain the same value is a restriction on the Cauchy data. If we use the above expressions (5.3), (5.4) to calculate $r$ derivatives of the iterates, then the requirement that they coincide leads to the same condition.

Differentiability of any finite order can be established along these lines if the coefficients of the equations are $C^{\infty}$. The Cauchy data have correspondingly to satisfy the appropriate comer conditions.

\section{Global solutions}

It is now fairly easy to combine our local result near the boundary with standard existence theorems to obtain a global statement. First we have to show that solutions of the constraint equations inside and outside the star do exist. Because of the decomposition into spherical harmonics the constraints are ordinary differential equations. Inside the star the functions $f, \dot{f}, g, \dot{g}, h, H, \dot{H}, W, V$ have to satisfy the equations (2.16), its time derivative, (2.7) and (2.8).

One way to satisfy all equations is the following: choose $f, \dot{f}, g, \dot{g}$ and $h$ as smooth functions such that the centre is regular and all functions have compact support within the star. Then we use (2.16) and its time derivative to define $H$ and $\dot{H}$. (Note that the linearized density $H$ need not be positive!) If we take vanishing data for all fields outside the star 
we have a global solution of the constraints which also satisfies all comer conditions at the boundary.

If one wants to prescribe the perturbed density one has to solve the constraints such that the geometrical quantities are regular at the centre.

We can also consider the case in which we take trivial data on the star and prescribe only data for a gravitational wave outside the star. In this case the functions $f, \dot{f}, g, \dot{g}$ and $h$ have to satisfy (2.16), its time derivative, $(2.7)$ and $(2.8)$ with vanishing matter variables. These are four equations for five functions, hence there seems to be only one free function. There is, however, the relation

$$
Z_{1}^{\prime}+Z_{1}-\dot{Z}-\frac{\lambda}{r^{2}} Z_{2}=0
$$

between the constraints. Hence two functions can be chosen arbitrarily.

From the equations it is not obvious that solutions with compact support exist. We can, however, take a linearized solution outside the star in any gauge and transform to ReggeWheeler gauge. If the solutions have spatially compact support in the original gauge, this will also be the case in Regge-Wheeler gauge because it is unique. To obtain such solutions we can, for example, use the superpotentials described in [10] or the Zerilli formalism [11].

Clearly, more solutions of the constraints can be constructed by similar considerations.

We have seen that there are many solutions of the constraints also satisfying the comer conditions at the boundary. The corresponding data determine a unique solution in some time interval $0 \leqslant t \leqslant T$. This solution can be constructed as follows: near the centre we use a general local theorem for linearized hydrodynamics [12] to get around the polar coordinate singularity of our equations; near the boundary we use the main result of this paper; for the remaining part we can use the well-known theorems for $1+1$ hyperbolic systems. As we take data of compact support we just have to use a finite number of overlapping patches to obtain a solution defined for $0 \leqslant t \leqslant T$.

The coefficients of the linear equations do not depend on time and are bounded as functions of $r$. Therefore we can extend the solution from $T$ to $2 T$ etc., hence it is defined for all $t$. The maximum of all our data can grow from $t=0$ to $t=T$ at most by a factor $q$, hence until time $n T$ by a factor $q^{n}$, which implies that the solution is exponentially bounded. This shows, in particular, that all solutions defined by data of compact support have Laplace transforms. The question under which conditions the solutions are bounded relates to the problem of stability which is not treated here.

Finally we would like to 'add the $Y_{l m}$ pieces' together. Suppose we have general data which can be decomposed into spherical harmonics. We obtain a solution for each $Y_{l m}$ contribution. We expect that the sum converges again. If this could be established-what we do not to intend here-one would have an existence theorem for fairly general data.

\section{References}

[1] Thorne K S and Campolattaro A 1967 Ap. J. 149591

[2] Lindbloom L and Detweiler S 1983 Ap. J. Suppl. 5373

[3] Chandrasekhar S and Ferrari V 1991 Proc. R. Soc. A 432247

[4] Kind S $1992 P h D$ Thesis LMU München

[5] Rendal] A D and Schmidt B G 1991 Class. Quantum Grav. 8985

[6] Synge J L 1960 Relativiry: The General Theory (Amsterdam: North-Holland)

[7] Courant R and Hilbert D 1962 Methods of Mathematical Physics (New York: Interscience)

[8] John F 1971 Partial Differential Equations (New York: Springer) 
[9] Nollert H-P and Schmidt B G 1992 Phys. Rev. D 452617

[10] Stewart J M 1979 Proc. Roy. Soc. A 367503

[11] Chandrasekhar S 1983 The Mathematical Theory of Black Holes (Oxford: Clarendon)

[12] Rendall A 1992 J. Math. Phys. 331047

[13] Kind S and Ehlers J 1993 Class. Quantum Grav. 10 2123-36 